\title{
Infecciones por enterobacterias productoras de betalactamasas de espectro extendido tras cirugía cardiaca: su impacto en la mortalidad
}

\author{
Francisco F. Rodríguez-Vidigal, Araceli Vera-Tomé, \\ Nieves Nogales-Muñoz y Agustín Muñoz-Sanz*
}

Servicio de Patología Infecciosa, Hospital Universitario de Badajoz, Servicio Extremeño de Salud, Universidad de Extremadura, Avenida de Elvas s/n, Badajoz, España

Recibido el 20 de marzo de 2015; aceptado el 13 de octubre de 2015

Disponible en Internet el 25 de noviembre de 2015

\section{PALABRAS CLAVE \\ Infección; \\ Cirugía cardiaca; \\ Epidemiología; \\ Mortalidad}

\begin{abstract}
Resumen
Introducción: Las enterobacterias productoras de betalactamasas de espectro extendido (EP BLEE) causan infecciones nosocomiales de modo creciente. Es controvertido si se asocian a peor pronóstico. El objetivo de este trabajo fue analizar si las infecciones por EP BLEE tras cirugía cardiaca presentan peor pronóstico que las causadas por enterobacterias no multirresistentes. Material y método: Estudio retrospectivo de las infecciones postquirúrgicas por enterobacterias, diagnosticadas en el Servicio de Cirugía Cardiaca de un Hospital Universitario (1/12/2007-1/12/2012). Se analizó la presencia de BLEE, la idoneidad del tratamiento empírico y la mortalidad global y relacionada.

Resultados: Se analizaron 61 pacientes $(67,2 \pm 10$ años). En 16 (26,2\%) se aislaron EP BLEE. Las especies más frecuentes fueron Escherichia coli (20 casos/9 BLEE), Enterobacter spp (18/1), Serratia marcescens (11/3), Proteus mirabilis (11/1) y Klebsiella spp (9/2). Las localizaciones más frecuentes fueron la sangre $(54,1 \%)$, las vías respiratorias $(31,1 \%)$ y la herida quirúrgica $(19,7 \%)$. El tratamiento empírico inicial fue no idóneo en mayor proporción en las infecciones por EP BLEE $(66,7 \%$ frente a $15,9 \%, \mathrm{p}<0,0001)$. Fallecieron 26 pacientes $(42,6 \%)$. La mortalidad global se asoció a infección por EP BLEE (odds ratio 5, 3; IC 95\% 1,3-21,5). La mortalidad atribuida a enterobacterias (14 pacientes) fue mayor cuando hubo bacteriemia ( $75 \%$ frente a $22 \%, p<0,02$ ) y el tratamiento empírico fue no idóneo (87,5\% frente a 43,7\%, p=0,05).

Conclusiones: La infección por EP BLEE en la post-cirugía cardiaca puede asociarse a mayor mortalidad, especialmente cuando hay bacteriemia. Ante la sospecha de infección post-quirúrgica por enterobacterias, se debe ajustar el tratamiento empírico según la incidencia local de EP BLEE.

(c) 2015 Sociedad Colombiana de Cardiología y Cirugía Cardiovascular. Publicado por Elsevier España, S.L.U. Este es un artículo Open Access bajo la licencia CC BY-NC-ND (http:// creativecommons.org/s/by-nc-nd/4.0/).
\end{abstract}

\footnotetext{
* Autor para correspondencia.

Correo electrónico: agus.munozsanz@gmail.com (A. Muñoz-Sanz).
} 


\section{KEYWORDS}

Infection;

Cardiac surgery;

Epidemiology;

Mortality
Extended-spectrum beta-lactamase-producing Enterobacteriaceae infections after cardiac surgery: their impact on mortality

\begin{abstract}
Introduction: Extended-spectrum beta-lactamase-producing Enterobacteriaceae (ES BLEE) increasingly cause nosocomial infections. It is controversial whether they are associated to a worse prognosis. The motivation for this study is to analyse if infections caused by ES BLEE after a cardiac surgery show a worse diagnosis that those caused by non-multidrug-resistant enterobacteriaceae.

Material and methods: Retrospective study of postoperative infections caused by enterobacteriaceae, diagnosed at the Cardiac Surgery Department (1/12/2007-1/12/2012). The presence of BLEE, the adequacy of the empirical treatment and global and related mortality were analysed. Results: 61 patients were analysed (67.2 \pm 10 years). In 16 (26.2\%) ES BLEE were found. Most commonly found species were Escherichia coli (20 cases/9 BLEE), Enterobacter spp (18/1), Serratia marcescens (11/3), Proteus mirabilis (11/1) and Klebsiella spp (9/2). Most frequent locations were blood (54.1\%), respiratory tract (31.1\%) and surgical wound (19.7\%). Initial empirical treatment was not adequate in greater proportion in infections for ES BLEE $(66.7 \%$ versus $15.9 \%, p<0,0001) .26$ patients died $(42.6 \%)$. Global mortality was associated to an ES BLEE infection (odds ratio $5.3 ; \mathrm{Cl} 95 \%$ 1.3-21.5). Mortality attributed to enterobacteriaceae (14 patients) was higher when bacteremia was present $(75 \%$ versus $22 \%, p<0,02)$ and empirical treatment was not adequate $(87.5 \%$ versus $43.7 \%, p=0,05)$.

Conclusions: Infections caused by ES BLEE in the cardiac postoperative period can be associated to higher mortality, especially when there is bacteremia. In suspicion of postoperative enterobacteriaceae infection, empirical treatment must be adjusted according to the local incidence of ES BLEE

( $) 2015$ Sociedad Colombiana de Cardiología y Cirugía Cardiovascular. Published by Elsevier España, S.L.U. This is an open access article under the CC BY-NC-ND license (http:// creativecommons.org/licenses/by-nc-nd/4.0/).
\end{abstract}

\section{Introducción}

Las enterobacterias productoras de las betalactamasas de espectro extendido (EP BLEE) causan las infecciones nosocomiales de un modo creciente en todo el mundo ${ }^{1-3}$. Es un asunto controvertido dilucidar si las infecciones por EP BLEE se asocian a un peor pronóstico. En un metaanálisis publicado en el año $2012^{4}$, se encuentra que las bacteriemias por EP BLEE se asocian a una mayor mortalidad que las causadas por las enterobacterias no multirresistentes, si bien esta asociación parece estar mediada por un tratamiento empírico inadecuado. Las bacteriemias por EP BLEE reciben un tratamiento empírico inapropiado con más frecuencia, incluso en estudios donde no se observa relación con la mortalidad ${ }^{5}$. En el ámbito concreto de las infecciones en pacientes sometidos a la cirugía cardiaca, existen pocos datos referidos al pronóstico de las infecciones por EP BLEE (búsqueda en PubMed usando los términos cardiac surgery y extended-spectrum betalactamase infection). Se ha descrito un brote de infecciones nosocomiales por Klebsiella pneumoniae productora de BLEE en pacientes pediátricos tras la cirugía cardiaca ${ }^{6}$ y se ha publicado un brote por Enterobacter cloacae, recogido en una unidad de cuidados intensivos cardiotorácica de adultos ${ }^{7}$. Nuestro objetivo fue analizar si las infecciones postquirúrgicas por EP BLEE en un servicio de cirugía cardiaca presentan un peor pronóstico que las causadas por las enterobacterias no multirresistentes, incluyendo tanto las bacteriemias como las infecciones de otra localización.

\section{Material y métodos}

Se estudiaron retrospectivamente, durante un período de cinco años (1/12/2007 a 1/12/2012), las infecciones postquirúrgicas por las enterobacterias diagnosticadas en el servicio de Cirugía Cardiaca del Complejo Hospitalario Universitario de Badajoz. Se trata de un centro de 880 camas, de referencia para una población de 1.108 .000 habitantes. El Servicio de Cirugía Cardiaca cuenta con una unidad de cuidados críticos postcirugía cardiaca (UCP), propia y ajena a la $\mathrm{UCl}$, y un área de hospitalización convencional. Para recoger todas las infecciones por las enterobacterias se revisaron los informes clínicos de la Unidad de Patología Infecciosa, que atiende a los pacientes hospitalizados en dicho servicio con sospecha de infección postquirúrgica, y se contrastaron con los aislados del Servicio de Microbiología. Se recogieron solo los casos de infección con actividad clínica, no las colonizaciones. El seguimiento de cada paciente duró hasta el alta hospitalaria.

Variables analizadas: 1) Edad, sexo y año de diagnóstico. 2) Tipo de intervención quirúrgica. 3) Especie de enterobacteria y presencia o no de las betalactamasas de espectro extendido (BLEE). Las BLEE fueron identificadas por microdilución con sistemas automatizados (Microscan, Siemens) $y$, en todos los casos, confirmadas a continuación mediante Etest (Liofilchem, Italia). 4) Localización de la infección por la enterobacteria, la coinfección o no por otras bacterias (gramnegativas no fermentadoras o grampositivas) y la presencia o no de candidemia. 5) Tratamiento antimicrobiano e 
idoneidad del tratamiento empírico (adecuación del tratamiento antimicrobiano empírico al patrón de susceptibilidad de la cepa bacteriana aislada). 6) Mortalidad intrahospitalaria global y mortalidad atribuida a la infección por las enterobacterias.

Se definió la bacteriemia por el aislamiento de las enterobacterias en, al menos, una de seis botellas de hemocultivos. Se definió la neumonía por: la aparición de un nuevo infiltrado en la radiografía (o en la tomografía computarizada) de tórax, el aislamiento de una enterobacteria en el aspirado traqueal, y al menos dos de los siguientes cuatro criterios: fiebre, leucocitosis o leucopenia, secreciones respiratorias purulentas y deterioro de la situación ventilatoria.

La infección de la herida quirúrgica vino definida por la presencia de signos inflamatorios locales y la emisión de exudado purulento (o dehiscencia de la sutura con exudación purulenta), donde se aisló la enterobacteria. La infección de escara se definió, así mismo, en virtud de los signos inflamatorios locales junto con la exudación purulenta. El diagnóstico de infección de vías urinarias se basó en la presencia de leucocituria y bacteriuria significativa $\left(\geq 10^{5}\right.$ unidades formadoras de colonias $/ \mu \mathrm{L}$ ) por una enterobacteria. Se consideró que la causa de la muerte era la infección por la enterobacteria (mortalidad atribuida) cuando concurrían: 1) aislamiento de la enterobacteria en sangre y/o en aspirado traqueal, 2) presencia de shock séptico, y 3) ausencia de otras causas concomitantes de muerte (shock cardiogénico, sepsis por otros microorganismos y complicación vascular cerebral). Se analizó la asociación de EP BLEE y de otros factores (tipo de intervención, localización de la infección, coinfección por otros microorganismos e idoneidad del tratamiento empírico) con la mortalidad global y atribuida.

Estudio estadístico. Para comparar las variables cuantitativas se utilizaron: la prueba de la t de Student y la prueba de Mann Whitney, según siguieran una distribución normal o no, respectivamente. Para las variables categóricas se empleó la prueba de chi al cuadrado o el test exacto de Fisher. Para detectar factores que influyeran en la mortalidad se llevó a cabo un análisis de regresión logística bivariado. Con posterioridad, se introdujeron en un modelo multivariado, personalizado, en pasos sucesivos hacia delante, las variables con diferencias con $\mathrm{p}<0,20$ en el análisis bivariado, ajustadas a la edad. Se consideró la diferencia estadísticamente significativa en los valores de $p$ inferior a 0,05. Todos los análisis estadísticos se llevaron a cabo con Statistical Package for the Social Sciences (SPSS) para Windows (versión 15,0; SPSS Inc., Chicago, IL, EE.UU., 2006).

\section{Resultados}

\section{Características de los pacientes y tipo de intervención quirúrgica}

Se diagnosticó la infección postcirugía cardiaca por las enterobacterias a 61 pacientes, de los cuales $35(57,5 \%)$ eran varones. Durante el período de estudio se llevaron a cabo 1.374 intervenciones con circulación extracorpórea; por tanto, la tasa de infección intrahospitalaria por las enterobacterias en pacientes sometidos a la cirugía cardiaca fue de 4,44\%. La media de edad fue de 67,2 \pm 10 años (mediana

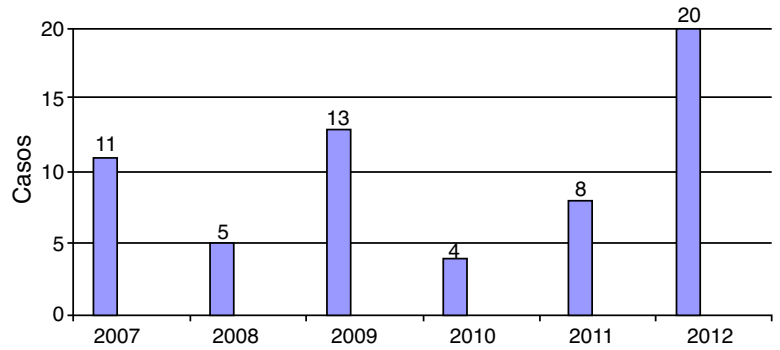

Figura 1 Distribución por años de los casos de infecciones por enterobacterias.

69, rango intercuartil 61-74). La fig. 1 muestra la distribución de casos por año. Todos los pacientes habían recibido antibioterapia profiláctica con la cefazolina, con excepción de los pacientes alérgicos a los betalactámicos, que recibieron la vancomicina.

La intervención quirúrgica consistió en cirugía de derivación aortocoronaria en 25 casos (41\%) y en sustitución valvular cardiaca en otros 25 casos (11 válvula mitral, 10 válvula aórtica y 4 doble sustitución mitral y aórtica). En siete pacientes $(11,5 \%)$ la indicación quirúrgica fue disección de la aorta torácica, en dos pacientes comunicación interventricular, en uno mixoma auricular y en uno trombo intraauricular. Desde el ingreso hasta la aparición de los síntomas de la infección trascurrió una mediana de 10 días (rango intercuartil, 4-20); solo un paciente padeció la infección postquirúrgica antes de las 48 horas de la cirugía. En ninguno de los casos existía evidencia de infección activa previa a la intervención quirúrgica.

\section{Infecciones por gramnegativos: etiología y localización}

Las especies de enterobacterias aisladas, así como los aislamientos concomitantes de otros microorganismos, se recogen en la tabla 1 . En $16 / 61$ aislados $(26,2 \%)$ se detectaron BLEE (9 E. coli, 3 S. marcescens, 2 Klebsiella pneumoniae, 1 Enterobacter cloacae y 1 P. mirabilis). La proporción de BLEE fue significativamente mayor en $E$. coli $(p<0,04)$. La localización de la infección por las enterobacterias fue: bacteriemia 33 casos (54,1\%), neumonía 19 $(31,1 \%)$, infección de la herida quirúrgica $12(19,7 \%)$, infección de vías urinarias $8(13,1 \%)$ e infección de escara 6 $(9,8 \%)$. Los focos de las bacteriemias fueron: bacteriemia primaria en 16 casos $(48,5 \%)$, respiratorio $7(21,2 \%)$, piel y partes blandas $5(15,1 \%)$, catéter $4(12,1 \%)$ y orina $1(3 \%)$.

\section{Tratamiento antimicrobiano y factores asociados con la mortalidad}

Los antimicrobianos empleados con mayor frecuencia para el tratamiento de la infección por las enterobacterias fueron el imipenem (68\%) y los aminoglucósidos (51\%). Con menor frecuencia se utilizaron la ceftazidima (26\%), la ceftriaxona (26\%), la piperacilina-tazobactam $(10,5 \%)$, el ciprofloxacino $(10,5 \%)$, el cefepime $(1,7 \%)$, la colistina $(1,7 \%)$ y la tigeciclina $(1,7 \%)$. El tratamiento empírico inicial fue inadecuado en 17 pacientes $(28,8 \%)$. Dicha circunstancia fue significativamente, más frecuente en los pacientes con la infección 
Tabla 1 Infecciones por enterobacterias y coinfecciones por otros microorganismos

\begin{tabular}{|c|c|c|}
\hline \multirow{2}{*}{$\frac{\text { Microorganismos }}{\text { Enterobacterias }}$} & \multicolumn{2}{|c|}{ Número de casos (\%) } \\
\hline & 61 & -100 \\
\hline Escherichia coli & 20 & $(32,8)$ \\
\hline Enterobacter spp & 18 & $(29,5)$ \\
\hline Serratia marcescens & 11 & $(18,0)$ \\
\hline Proteus mirabilis & 11 & $(18,0)$ \\
\hline Klebsiella spp & 9 & $(14,7)$ \\
\hline Morganella morganii & 3 & $(4,9)$ \\
\hline Citrobacter spp & 2 & $(3,3)$ \\
\hline \multicolumn{3}{|l|}{ Otros microorganismos } \\
\hline $\begin{array}{l}\text { Bacilos gramnegativos no } \\
\text { fermentadores }\end{array}$ & 9 & $(14,7)$ \\
\hline Pseudomonas & 6 & \\
\hline \multicolumn{3}{|l|}{ aeruginosa } \\
\hline \multicolumn{3}{|l|}{ maltophilia } \\
\hline \multicolumn{2}{|l|}{ baumannii } & \\
\hline Cocos grampositivos & 34 & $(55,7)$ \\
\hline Staphylococcus & 18 & $(29,5)$ \\
\hline \multicolumn{3}{|l|}{ coagulasa negativo } \\
\hline Enterococcus spp & 13 & $(21,3)$ \\
\hline Staphylococcus aureus & 9 & $(14,7)$ \\
\hline Streptococcus spp & 4 & $(6,5)$ \\
\hline Candida spp. & 5 & $(8,2)$ \\
\hline
\end{tabular}

por EP BLEE que en aquellos con enterobacterias no productoras de BLEE (10/15 -66,7\%- frente a 7/44 -15,9\%-, $\mathrm{p}<0,0001$ ).

Durante el ingreso hospitalario fallecieron 26 pacientes $(42,6 \%)$, en $14(22,9 \%)$, la muerte se atribuyó a la infección por las enterobacterias (shock séptico con bacteriemia y/o neumonía por enterobacteria). Entre los pacientes fallecidos hubo significativamente más enfermos con infección por EP BLEE que entre los supervivientes (42,4\% -11/26frente a 14,3\% -5/35-, $p=0,015)$ y mayor proporción de disección aórtica como indicación quirúrgica (25,0\% -6/24frente a 3,0\% -1/33-, $p=0,034)$. El 68,7\% de los pacientes con infección por EP BLEE (11 de 16) murió. No hubo diferencias respecto a la edad, el sexo, la coinfección por cocos grampositivos, la candidemia y la idoneidad del tratamiento antimicrobiano empírico (tabla 2). En el análisis multivariante, los únicos factores relacionados con la mortalidad global fueron: la infección por EP BLEE y la disección aórtica (tabla 3). Entre los pacientes fallecidos en relación con la infección por las enterobacterias, hubo mayor proporción de bacteriemia (85,7\% -12/14- frente a 36,4\% -4/7-, p=0,016) y menor proporción de terapia antimicrobiana empírica adecuada (50\% -7/14- frente a 90\% -1/10-, $p=0,051)$ (tabla 4).

\section{Discusión}

El presente estudio revela que la infección postquirúrgica por EP BLEE en un servicio de cirugía cardiaca puede tener un protagonismo relevante en cuanto a la frecuencia y que puede asociarse a una mayor mortalidad intrahospitalaria. Además, proporciona apoyo para incluir una cobertura anti-
Tabla 2 Factores asociados con la mortalidad intrahospitalaria global

\begin{tabular}{|c|c|c|c|}
\hline Factor & $\begin{array}{l}\text { Fallecidos ( } 26 \\
\text { pacientes) \% }\end{array}$ & $\begin{array}{l}\text { Supervivientes } \\
\text { (35 pacientes) } \\
\%\end{array}$ & $\mathrm{p}$ \\
\hline Edad (años) & $68,1 \pm 10,6$ & $66,6 \pm 9,7$ & 0,56 \\
\hline Sexo varón & 61,5 & 54,3 & 0,38 \\
\hline $\begin{array}{r}\text { Disección } \\
\text { aórtica }\end{array}$ & 25,0 & 3,0 & $<0,04$ \\
\hline EP BLEE & 42,4 & 14,3 & $<0,02$ \\
\hline$B G N N F$ & 42,3 & 31,4 & 0,27 \\
\hline $\begin{array}{c}\text { Cocos gram- } \\
\text { positivos }\end{array}$ & 57,7 & 54,3 & 0,5 \\
\hline Candidemia & 11,4 & 2,9 & 0,1 \\
\hline $\begin{array}{l}\text { Antibiótico } \\
\text { empírico } \\
\text { apropiado }\end{array}$ & 68,0 & 73,5 & 0,43 \\
\hline
\end{tabular}

BGN NF: bacilos gramnegativos no fermentadores. EP BLEE: enterobacteria productora de betalactamasas de espectro extendido.

Las variables cuantitativas se expresan como media \pm desviación estándar. Las variables cualitativas están expresadas en porcentaje.

Tabla 3 Factores relacionados con la mortalidad global en el análisis multivariante

\begin{tabular}{lcl}
\hline Factor & Odds & $\begin{array}{l}\text { Intervalo de } \\
\text { confianza 95\% }\end{array}$ \\
\hline ratio & 23,2 & $2,1-253,2$ \\
Infección aórtica & 5,3 & $1,3-21,5$ \\
Candidemia & 9,3 & $0,8-101,1$ \\
\hline
\end{tabular}

EP BLEE: enterobacteria productora de betalactamasas de espectro extendido

microbiana empírica frente a las EP BLEE cuando se sospeche de infección por las enterobacterias (especialmente la bacteriemia) en los servicios quirúrgicos con elevada incidencia de infecciones por EP BLEE. Los pacientes de nuestra serie

Tabla 4 Factores asociados con la mortalidad atribuida a la infección por enterobacterias

\begin{tabular}{lllr}
\hline Factor & $\begin{array}{l}\text { Muerte } \\
\text { relacionada (14 } \\
\text { pacientes) \% }\end{array}$ & $\begin{array}{l}\text { No relacionada } \\
\text { (11 pacientes) \% }\end{array}$ & $\mathrm{p}$ \\
\hline Edad (años) & $67,0 \pm 13$ & $69,1 \pm 7,5$ & 0,54 \\
Sexo varón & 64,3 & 63,6 & 0,65 \\
EP BLEE & 50,0 & 36,4 & 0,39 \\
Bacteriemia & 85,7 & 36,4 & $<0,02$ \\
Candidemia & 7,1 & 27,3 & 0,21 \\
$\begin{array}{c}\text { Antibiótico } \\
\text { empírico }\end{array}$ & 50,0 & 90,0 & 0,051 \\
apropiado & & & \\
\hline
\end{tabular}

EP BLEE: enterobacteria productora de betalactamasas de espectro extendido. Las variables cuantitativas están expresadas como media \pm desviación estándar. Las variables cualitativas en porcentaje. 
presentan la peculiaridad de haber sido sometidos a procedimientos quirúrgicos de alto riesgo y de necesitar soporte postoperatorio en una unidad de cuidados críticos. De ahí la elevada mortalidad de las infecciones estudiadas, habitualmente acompañadas de shock séptico, y la alta frecuencia de coinfección por otros microorganismos.

Refiriéndonos solo a las infecciones por EP BLEE tras la realización de la cirugía cardiaca, hasta la fecha se han publicado casos aislados o brotes por: $K$. pneumoniae, $E$. coli y $E$. cloacae cl-10 $^{6}$. Según nuestra experiencia, se pueden añadir a la lista: S. marcescens (tres casos) y P. mirabilis.

Hemos detectado una elevada tasa de letalidad global (42\%), mayor para el subgrupo de pacientes con infección por EP BLEE $(68,7 \%)$. Estas cifras coinciden con las de Melzer et al. ${ }^{11}(61 \%)$ en pacientes con la bacteriemia por $E$. coli BLEE. Otros autores hallan una mortalidad menor (rango de 29-35\%) en infecciones por EP BLEE (frente a 11-24\% para las enterobacterias no BLEE) $)^{5,12-14}$. En todos estos estudios se analizan las bacteriemias por $E$. coli (a veces también por $K$. pneumoniae) que afectan a pacientes de servicios tanto médicos como quirúrgicos, o únicamente a personas con fiebre neutropénica. En nuestro caso, todos los enfermos habían tenido una intervención de cirugía cardiaca, con necesidad de soporte ventilatorio e inotropo y, en más de la mitad de los casos, coinfección por otras bacterias o candidemia. La infección por EP BLEE se asoció de modo independiente con la mortalidad global. El otro factor asociado fue el diagnóstico de disección aórtica como indicación quirúrgica, lo cual es un hecho acorde con el elevado riesgo y el habitual carácter urgente de dicho procedimiento quirúrgico. El amplio intervalo de confianza es una consecuencia del escaso número de pacientes (siete) con esta condición.

El tratamiento antimicrobiano empírico inicial fue inapropiado en el $\mathbf{2 8 , 8} \%$ de los casos. En un estudio multicéntrico español con 387 bacteriemias por: $E$. coli y $K$. pneumoniae $^{15}$, el tratamiento fue inadecuado en el $51,2 \%$ de los casos. En nuestra experiencia, la inadecuación terapéutica fue más frecuente en las infecciones por EP BLEE, un dato recogido antes por otros autores ${ }^{5,16}$. Es notable consignar que hubo una tendencia a la asociación de la mortalidad atribuida a la infección por las bacterias gramnegativas con la elección empírica de un antimicrobiano no eficaz frente a EP BLEE (sobre todo la ceftriaxona y la ceftazidima). Del mismo modo, en el trabajo de Peralta et al. ${ }^{15}$, un tratamiento empírico adecuado resultó protector frente a la mortalidad.

Un aspecto que vale la pena resaltar es la importancia de vigilar y prevenir las infecciones debidas a microorganismos multirresistentes en el ámbito hospitalario. En algunas unidades de cuidados intensivos que atienden a pacientes intervenidos del corazón, se ha reseñado una alta proporción de $E$. coli y de $K$. pneumoniae productoras de BLEE en frotis rectales de vigilancia epidemiológica ${ }^{17}$. En nuestro centro, el celo en la aplicación de medidas de control de superficies y material clínico y la concienciación del personal sanitario en la higiene de las manos, han contribuido a que, durante el año 2013, no se hayan detectado nuevos casos de infecciones por EP BLEE dentro del servicio de cirugía cardiaca.

Las limitaciones principales del estudio son: 1) el carácter retrospectivo y el escaso número de casos (61 pacientes), 2) la no inclusión de variables que reflejen la situación hemodinámica de los pacientes tras la cirugía, 3) el porcentaje importante de infecciones polimicrobianas (inevitable en pacientes postquirúrgicos hospitalizados en una unidad de críticos) y 4) la inclusión de infecciones por diferentes géneros de las enterobacterias (Escherichia spp, Serratia spp, Klebsiella spp, Enterobacter spp y Proteus spp). Como ventaja que puede atenuar las deficiencias citadas figura la homogeneidad de la muestra: todos los pacientes fueron intervenidos quirúrgicamente y recibieron cuidados intensivos e infectológicos en el mismo servicio hospitalario por el mismo equipo multidisciplinar (cirujano, anestesiólogo e infectólogo). Con respecto a la inclusión de géneros distintos de las enterobacterias, es congruente con la realidad biológica la coexistencia de todos ellos en la microbiota del hospedador y con los mecanismos comunes de transmisión horizontal de los genes responsables de la producción de $\mathrm{BLEE}^{18}$. Por otra parte, en otros estudios llevados a cabo con anterioridad, en los que se valoró la asociación de las infecciones por EP BLEE con la mortalidad, se han incluido las bacteriemias por $E$. coli y por K. pneumoniae ${ }^{12,19}$.

Concluimos reforzando la conveniencia de analizar regularmente el perfil de resistencias de las enterobacterias (BLEE) en cada servicio quirúrgico por separado, no de forma global. De igual modo, se recomienda el uso empírico de antimicrobianos eficaces frente a EP BLEE cuando se llevan a cabo intervenciones de alto riesgo en los servicios con alta incidencia de EP BLEE.

\section{Responsabilidades éticas}

Protección de personas y animales. Los autores declaran que para esta investigación no se han realizado experimentos en seres humanos ni en animales.

Confidencialidad de los datos. Los autores declaran que en este artículo no aparecen datos de pacientes.

Derecho a la privacidad y consentimiento informado. Los autores declaran que en este artículo no aparecen datos de pacientes.

\section{Conflicto de intereses}

Los autores declaran no tener ningún conflicto de intereses.

\section{Bibliografía}

1. Oteo J, Pérez-Vázquez M, Campos J. Extended-spectrum [beta]lactamase producing Escherichia coli: changing epidemiology and clinical impact. Curr Opin Infect Dis. 2010;23:320-6.

2. Van Duinj PJ, Dautzenberg MJ, Oostdijk EA. Recent trends in antibiotic resistance in European ICUs. Curr Opin Crit Care. 2011;17:658-65.

3. de Kraker ME, Jarlier V, Monen JC, Heuer OE, van de Sande $\mathrm{N}$, Grundmann H. The changing epidemiology of bacteraemias in Europe: trends from the European Antimicrobial Resistance Surveillance System. Clin Microbiol Infect. 2013;19:860-8.

4. Rottier WC, Ammerlaan HSM, Banten MJM. Effects of confounders and intermediates on the association of bacteraemia caused by extended-spectrum b-lactamase - producing Enterobacteriaceae and patient outcome: a meta-analysis. J Antimicrob Chemother. 2012;67:1311-20. 
5. García-Hernández A, García-Vázquez E, Gómez-Gómez J, Canteras M, Hernández-Torres A, Ruiz-Gómez J. Bacteriemia por Escherichia coli: factores predictivos de presencia de bacterias productoras de betalactamasas de espectro extendido e influencia de la resistencia en la mortalidad de los pacientes. Med Clin (Barc). 2011;136:56-60.

6. French GL, Shanoon KP, Simmons N. Hospital outbreak of Klebsiella pneumoniae resistant to broad-sprectrum cephalosporins and beta-lactam-beta-lactamase inhibitor combinations by hyperproduction of SHV-5 beta-lactamase. J Clin Microbiol. 1996;34:358-63.

7. Manzur A, Tubau F, Pujol M, Calatayud L, Domínguez MA, Peña C, et al. Nosocomial outbreack due to extended-spectrum-betalactamase-producing Enterobacter cloacae in a cardiothoracic intensive care unit. J Clin Microbiol. 2007;45:2365-9.

8. Tan L, Sun X, Zhu X, Zhang Z, Li J, Shu Q. Epidemiology of nosocomial pneumonia in infants after cardiac surgery. Ches. 2004;125:410-7.

9. Takahashi Y, Tsutsumi Y, Monta O, Chashi H. Mycotic aneurysm of the thoracic aorta caused by extended-spectrum betalactamase-producing Escherichia coli. Interact Cardiovasc Thorac Surg. 2011;12:61-2.

10. Valenzuela H, Carrascal Y, Maroto L, Arce N. Fulminant mediastinitis due to extended-spectrum beta-lactamase-producing Klebsiella pneumoniae: atypical presentation and spreeading following cardiac surgery. Interact Cardiovasc Thorac Surg. 2013;16:703-4.

11. Melzer M, Petersen I. Mortality following bacteraemic infection caused by extended spectrum beta-lactamase (ESBL) producing $E$. coli compared to non-ESBL producing $E$. coli. J Infect. 2007;55:254-9.

12. Lee $\mathrm{Cl}$, Lee NY, Yan JJ, Lee HC, Ko NY, Chang CM, et al. Extended-spectrum beta-lactamase-producing phenotype signifies a poor prognosis for patients with cefpodoxime-resistant Escherichia coli or Klebsiella pneumoniae bacteremia. J Microbiol Immunol Infect. 2009;42:303-9.
13. Cornejo-Juárez P, Pérez-Jiménez C, Silva-Sánchez J, VelázquezAcosta C, González-Lara F, Reyna-Flores E, et al. Molecular analysis and risk factors for Escherichia coli producing extended-spectrum beta-lactamase bloodstream infection in hematological malignancies. PLoS One. 2012;7(4):e35780, http://dx.doi.org/10.1371/journal.pone.0035780.

14. Anunnatsiri S, Towiwat P, Chaimanee P. Risk factors and clinical outcomes of extended spectrum beta-lactamase (ESBL)producing Escherichia coli septicemia at Srinagarind University Hospital, Thailand. Southeast Asian J Trop Med Public Health. 2012;43:1169-77.

15. Peralta G, Lamelo $M$, Álvarez-García $P$, Velasco $M$, Delgado A, Horcajada JP, et al. Impact of empirical treatment in extended-spectrum beta-lactamase-producing Escherichia coli and Klebsiella spp. bacteriemia. A multicentric cohort study. BMC Infect Dis. 2012;12:245, http://dx.doi.org/10.1186/1471-2334-12-245.

16. Lin JN, Chen YH, Chang LL, Lai CH, Lin HL, Lin HH. Clinical characteristics and outcomes of patients with extended-spectrum beta-lactamase-producing bacteremias in the emergency department. Intern Emerg Med. 2011;6:547-55.

17. Vasques MR, Bello AR, Lamas C, da C, Correa J, Pereira JA. Beta-lactamase producing enterobacteria isolated from surveillance swabs of patients in a cardiac intensive care unit in Rio de Janeiro, Brazil. Braz J Infect Dis. 2011;15: 28-33.

18. Doi Y, Adams-Haduch JM, Peleg AY, D'Agata EM. The role of horizontal gene transfer in the dissemination of extended-spectrum beta-lactamase-producing Escherichia coli and Klebsiella pneumoniae isolates in an endemic setting. Diagn Microbiol Infect Dis. 2012;74:34-8.

19. Kim SH, Kwon JC, Choi SM, Lee DG, Park SH, Choi JH, et al. Escherichia coli and Klebsiella penumoniae bacteremia in patients with neutropenic fever: factors associated with extended-spectrum beta-lactamase production and its impact on outcome. Ann Hematol. 2013;92:533-41. 